\title{
Non-Polynomial Spline Method for a Time-Dependent Heat-Like Lane- Emden Equation
}

\author{
S.H. CAGlar AND M.F. UCAR* \\ Department of Mathematics - Computer, Istanbul Kultur University, 34156 Atakoy, Istanbul, Turkey \\ In this study, a time-dependent heat-like Lane-Emden equation is solved by using a non-polynomial spline \\ method. An example is solved to assess the accuracy of the method. The numerical results are obtained for \\ different values $(n)$ of equation. The results indicate that non-polynomial spline method is effectively implemented. \\ It is seen that results are compatible with exact solutions and consistent with other existing numerical methods.
}

PACS: 02.30.Jr, 02.60.Lj, 02.70.Bf, 02.70.Jn, 44.05.+e

\section{Introduction}

In this paper, we consider heat-type equation for physical problems

$$
u_{x x}+\frac{r}{x} u_{x}+\operatorname{ag}(x, t) y(u)+h(x, t)=u_{t},
$$

for $0<x \leq L, 0<t<T, r>0, a \in Z$, subject to the boundary conditions $u(0, t)=v(t)$, and $u_{x}(0, t)=0$ where $g(x, t) y(u)+h(x, t)$ is nonlinear heat source, $u(x, t)$ is the temperature, and $t$ is the dimensionless time variable.

Some researchers dealed with this type of models [1-9]. These physical models describe some important equations as the Navier-Stokes equations can be converted into various heat-like equation in some special cases [10]. Furthermore, thermo-pore-elastic equations describing fluid migration through fluid-saturated porous media at depth in the crust [11] and this theory [12] can be expressed in heat-like equations. The analytic solutions to several forms of the above problem were presented by Shawagfeh [4], Wazwaz [13-16] using the Adomian decomposition method. Chowdhury [17] and Hashim [18], He et al. [19-21] have solved these problems with using homotopy-perturbation and variational iterational methods, Momani [22] applied the method to the time fractional heat-like equation with variable coefficient.

In this paper, we solved this problem numerically with non-polynomial spline method and found absolute errors which are obtained by comparing numerical results with existing above analytic (exact) solutions. The paper is organized as follows: non-polynomial spline method is described in Sect. 2 briefly. In Sect. 3 the method of solution of Eq. (1) is presented. In Sect. 4 some numerical result that are illustrated using Matlab 7.0 are given to clarify the method. Concluding remarks are given in Sect. 5 .

\footnotetext{
* corresponding author; e-mail: f.ucar@iku.edu.tr
}

\section{Non-polynomial spline method}

We divide the interval $[a, b]$ into $n$ equal subintervals using the grid points $x_{i}=a+i h, i=0,1,2, \ldots, n$ with $a=x_{0}, x_{n}=b, h=(b-a) / n$, where $n$ is an arbitrary positive integer. Let $u(x)$ be the exact solution and $u_{i}$ be an approximation to $u\left(x_{i}\right)$ obtained by the non-polynomial cubic $S_{i}(x)$ passing through the points $\left(x_{i}, u_{i}\right)$ and $\left(x_{i+1}, u_{i+1}\right)$, we do not only require that $S_{i}(x)$ satisfies interpolatory conditions at $x_{i}$ and $x_{i+1}$, but also the continuity of first derivative at the common nodes $\left(x_{i}, u_{i}\right)$ are fulfilled. We write $S_{i}(x)$ in the form

$$
\begin{aligned}
& S_{i}(x)=a_{i}+b_{i}\left(x-x_{i}\right)+c_{i} \sin \tau\left(x-x_{i}\right) \\
& \quad+d_{i} \cos \tau\left(x-x_{i}\right), \quad i=0,1, \ldots, n-1,
\end{aligned}
$$

where $a_{i}, b_{i}, c_{i}$, and $d_{i}$ are constants and $\tau$ is a free parameter. A non-polynomial function $S(x)$ of class $C^{2}[a, b]$ interpolates $u(x)$ at the grid points $x_{i}, i=0,1,2, \ldots, n$, depends on a parameter $\tau$, and reduces to ordinary cubic spline $S(x)$ in $[a, b]$ as $\tau \rightarrow 0$.

To derive expression for the coefficients of Eq. (2) in term of $u_{i}, u_{i+1}, M_{i}$ and $M_{i+1}$, we first define:

$$
\begin{aligned}
& S_{i}\left(x_{i}\right)=u_{i}, \quad S_{i}\left(x_{i+1}\right)=u_{i+1}, \\
& S_{i}^{\prime \prime}\left(x_{i}\right)=M_{i}, \quad S_{i}^{\prime \prime}\left(x_{i+1}\right)=M_{i+1} .
\end{aligned}
$$

From algebraic manipulation, we get the following expression:

$$
\begin{aligned}
& a_{i}=u_{i}+\frac{M_{i}}{\tau^{2}}, \quad b_{i}=\frac{u_{i+1}-u_{i}}{h}+\frac{M_{i+1}-M_{i}}{\tau \theta}, \\
& c_{i}=\frac{M_{i} \cos \theta-M_{i+1}}{\tau^{2} \sin \theta}, \quad d_{i}=-\frac{M_{i}}{\tau^{2}},
\end{aligned}
$$

where $\theta=\tau h$ and $i=0,1,2, \ldots, n-1$.

Using the continuity of the first derivative at $\left(x_{i}, u_{i}\right)$, that is $S_{i-1}^{\prime}\left(x_{i}\right)=S_{i}^{\prime}\left(x_{i}\right)$ we obtain the following relations for $i=1, \ldots, n-1$ :

$$
\alpha M_{i+1}+2 \beta M_{i}+\alpha M_{i-1}=\left(1 / h^{2}\right)\left(u_{i+1}-2 u_{i}+u_{i-1}\right),
$$

where $\alpha=-1 / \theta^{2}+1 / \theta \sin \theta, \beta=-1 / \theta^{2}-\cos \theta / \theta \sin \theta$ and $\theta=\tau h$. 
The method is fourth-order convergent if $1-2 \alpha-2 \beta=0$ and $\alpha=1 / 12$ [23].

\section{Analysis of method}

To illustrate the application of the spline method developed in the previous section we consider time-dependent heat-like Lane-Emden equation that is given in Eq. (1).

At the grid point $\left(x_{i}, u_{i}\right)$, the proposed problem in Eq. (1) may be discretized by

$$
u_{i}^{\prime \prime}+\frac{r}{x_{i}} u_{i}^{\prime}+a g\left(x_{i}, t\right) u_{i}+h\left(x_{i}, t\right)=\frac{u_{i}-u_{i-1}}{k} .
$$

Substituting $M_{i}=u_{i}^{\prime \prime}$ and $f\left(x_{i}\right)=u_{i-1}$ in Eq. (5), we get

$$
M_{i}+\frac{r}{x_{i}} u_{i}^{\prime}+\operatorname{ag}\left(x_{i}, t\right) u_{i}+h\left(x_{i}, t\right)=\frac{u_{i}-f\left(x_{i}\right)}{k} .
$$

Solving Eq. (6) for $M_{i}$, we obtain

$$
M_{i}=\left(\frac{1}{k}-a g\left(x_{i}, t\right)\right) u_{i}-\frac{r}{x_{i}} u_{i}^{\prime}-h\left(x_{i}, t\right)-\frac{f\left(x_{i}\right)}{k} .
$$

The following approximations for the first-order derivative of $u$ in Eq. (7) can be used:

$$
\begin{aligned}
& u_{i}^{\prime}=\frac{u_{i+1}-u_{i-1}}{2 h}, \quad u_{i+1}^{\prime}=\frac{3 u_{i+1}-4 u_{i}+u_{i-1}}{2 h}, \\
& u_{i-1}^{\prime}=\frac{-u_{i+1}+4 u_{i}-3 u_{i-1}}{2 h} .
\end{aligned}
$$

Therefore Eq. (7) becomes

$$
\begin{aligned}
M_{i} & =\left(\frac{1}{k}-\operatorname{ag}\left(x_{i}, t\right)\right) u_{i}-\frac{r}{x_{i}}\left(\frac{u_{i+1}-u_{i-1}}{2 h}\right) \\
& -h\left(x_{i}, t\right)-\frac{f\left(x_{i}\right)}{k}
\end{aligned}
$$

and

$$
\begin{aligned}
& M_{i+1}=\left(\frac{1}{k}-a g\left(x_{i+1}, t\right)\right) u_{i+1}-\frac{r}{x_{i+1}}\left(\frac{3 u_{i+1}-4 u_{i}+u_{i-1}}{2 h}\right) \\
& -h\left(x_{i+1}, t\right)-\frac{f\left(x_{i+1}\right)}{k}, \\
& M_{i-1}=\left(\frac{1}{k}-a g\left(x_{i-1}, t\right)\right) u_{i-1}-\frac{r}{x_{i-1}}\left(\frac{-u_{i+1}+4 u_{i}-3 u_{i-1}}{2 h}\right) \\
& -h\left(x_{i-1}, t\right)-\frac{f\left(x_{i-1}\right)}{k} .
\end{aligned}
$$

Substituting Eqs. (9)-(11) in Eq. (4), we find the following $n-1$ linear algebraic equations in the $n+1$ unknowns for $i=0,1, \ldots, n$ :

$$
\begin{aligned}
& {\left[\alpha\left(\frac{1}{k}-a g\left(x_{i+1}, t\right)-\frac{3}{x_{i+1} h}+\frac{1}{x_{i-1} h}\right)\right.} \\
& \left.\quad-\frac{2 \beta}{x_{i} h}-\frac{1}{h^{2}}\right] u_{i+1}+\left[4 \alpha\left(\frac{1}{x_{i+1} h}-\frac{1}{x_{i-1} h}\right)\right. \\
& \left.+2 \beta\left(\frac{1}{k}-a g\left(x_{i}, t\right)\right)+\frac{2}{h^{2}}\right] u_{i}\left[\alpha \left(\frac{1}{k}-a g\left(x_{i-1}, t\right)\right.\right. \\
& \left.\left.-\frac{1}{x_{i+1} h}+\frac{3}{x_{i-1} h}\right)+\frac{2 \beta}{x_{i} h}-\frac{1}{h^{2}}\right] u_{i-1} \\
& -\frac{1}{k}\left(\alpha f\left(x_{i+1}\right)+2 \beta f\left(x_{i}\right)+\alpha f\left(x_{i-1}\right)\right) \\
& -k\left[\alpha h\left(x_{i+1}\right)+2 \beta h\left(x_{i}\right)+\alpha h\left(x_{i-1}\right)\right]=0 .
\end{aligned}
$$

We need two more equations. The two end conditions can be derived as follows:

$$
u(0, t)=v(t), \quad u(1, t)=z(t) .
$$

Substituting

$$
\begin{aligned}
& c 1=\alpha\left(\frac{1}{k}-a g\left(x_{i-1}, t\right)-\frac{1}{x_{i+1} h}+\frac{3}{x_{i-1} h}\right)+\frac{2 \beta}{x_{i} h}-\frac{1}{h^{2}}, \\
& c 2=4 \alpha\left(\frac{1}{x_{i+1} h}-\frac{1}{x_{i-1} h}\right)+2 \beta\left(\frac{1}{k}-a g\left(x_{i}, t\right)\right)+\frac{2}{h^{2}}, \\
& c 3=\alpha\left(\frac{1}{k}-a g\left(x_{i+1}, t\right)-\frac{3}{x_{i+1} h}+\frac{1}{x_{i-1} h}\right)-\frac{2 \beta}{x_{i} h}-\frac{1}{h^{2}},
\end{aligned}
$$

we get these matrix forms

$$
A=\left[\begin{array}{ccccccc}
1 & 0 & 0 & 0 & \ldots & 0 & 0 \\
c 1 & c 2 & c 3 & 0 & \ldots & 0 & 0 \\
0 & c 1 & c 2 & c 3 & 0 & \ldots & 0 \\
. & . & . & . & . & . & . \\
. & . & . & . & . & . & . \\
. & . & . & . & . & . & . \\
0 & . & 0 & 0 & c 1 & c 2 & c 3 \\
. & . & . & . & 0 & 0 & 1
\end{array}\right],
$$

$$
B=\left[\begin{array}{c}
v(t) \\
\frac{1}{k}\left[\alpha f\left(x_{2}\right)+2 \beta f\left(x_{1}\right)+\alpha f\left(x_{0}\right)\right]+k\left[\alpha h\left(x_{2}\right)+2 \beta h\left(x_{1}\right)+\alpha h\left(x_{0}\right)\right] \\
\frac{1}{k}\left[\alpha f\left(x_{3}\right)+2 \beta f\left(x_{2}\right)+\alpha f\left(x_{1}\right)\right]+k\left[\alpha h\left(x_{3}\right)+2 \beta h\left(x_{2}\right)+\alpha h\left(x_{1}\right)\right] \\
\cdot \\
\cdot \\
\cdot \\
\frac{1}{k}\left[\alpha f\left(x_{n}\right)+2 \beta f\left(x_{n-1}\right)+\alpha f\left(x_{n-2}\right)\right]+k\left[\alpha h\left(x_{n}\right)+2 \beta h\left(x_{n-1}\right)+\alpha h\left(x_{n-2}\right)\right]
\end{array}\right],
$$




$$
U=\left[u_{0}, u_{1}, \ldots, u_{n}\right]^{\prime} .
$$

Finally the approximate solution is obtained by solving $A U=B$ using Matlab 7.0.1.

\section{Numerical example}

In this section, we test our scheme on an example. We consider the numerical results obtained by applying the scheme discussed above to the following equation:

$$
\begin{gathered}
u^{\prime \prime}+\frac{2}{x} u^{\prime}-\left(6+4 x^{2}-\cos t\right) u=u_{t}, \\
0<x<1, \quad t>0,
\end{gathered}
$$

with initial condition $u(x, 0)=\mathrm{e}^{x^{2}}$, and boundary conditions $u(0, t)=\mathrm{e}^{\sin t}, u_{x}(0, t)=0$. The exact solution of the above problem is $u(x, t)=\mathrm{e}^{x^{2}+\sin t}$. The problem is solved by using the scheme (13) in this paper. The maximum absolute errors are listed in Table.

TABLE

Maximum absolute errors of the scheme (13).

\begin{tabular}{c|c|c|c}
\hline \hline$n$ & $k=0.1$ & $k=0.01$ & $k=0.001$ \\
\hline 11 & $2.3314 \times 10^{-2}$ & $1.2794 \times 10^{-2}$ & $6.8863 \times 10^{-3}$ \\
21 & $5.8066 \times 10^{-3}$ & $4.1258 \times 10^{-3}$ & $1.9090 \times 10^{-3}$ \\
41 & $3.1664 \times 10^{-3}$ & $1.4558 \times 10^{-3}$ & $7.8276 \times 10^{-4}$ \\
61 & $1.1522 \times 10^{-3}$ & $7.8052 \times 10^{-4}$ & $5.9650 \times 10^{-4}$ \\
121 & $8.0058 \times 10^{-4}$ & $1.9542 \times 10^{-4}$ & $5.2565 \times 10^{-5}$
\end{tabular}

\section{Conclusion}

In this paper non-polynomial spline method is applied for the numerical solution of the heat-like time dependent Lane-Emden equation and the maximum absolute errors have been shown in Table, which shows that this method approximates the exact solution very well. The implementation of the present method is more computational than the existing methods.

\section{References}

[1] J.R. Holiday, J.B. Rundle, K.F. Tiampo, Tectonophysics 413, 87 (2006).

[2] A. Goruis, Appl. Math. Comput. 173, 126 (2006).

[3] H.T. Davis, Introduction to Nonlinear Differential and Integral Equations, Dover, New York 1962.

[4] N.T. Shawagfeh, J. Math. Phys. 34, 4364 (1993).

[5] A.M. Wazwaz, Appl. Math. Comput. 166, 638 (2005).

[6] J.-H. He, Int. J. Non-Linear Mech. 34, 699 (1999).

[7] S. Momani, Appl. Math. Comput. 165, 459 (2005).

[8] A.S. Bataineh, M.S.M. Noorani, I. Hashim, Phys. Lett. A 371, 72 (2007).

[9] B. Batiha, M.S.M. Noorani, Phys. Lett. A 369, 55 (2007).

[10] R.H. Nochetto, J.H. Pyo, Math. Comp. 74, 521 (2005).

[11] A.L. Merlani, G. Natale, E. Salusti, Geophys. Astrophys. Fluid Dyn. 85, 97 (1997).

[12] G. Natale, Pure Appl. Geophys. 152, 193 (1998).

[13] A.M. Wazwaz, Appl. Math. Comput. 118, 287 (2001).

[14] A.M. Wazwaz, Appl. Math. Comput. 128, 45 (2002).

[15] A.M. Wazwaz, Appl. Math. Comput. 173, 165 (2006).

[16] A.M. Wazwaz, Appl. Math. Comput. 161, 543 (2005).

[17] M.S.H. Chowdhury, I. Hashim, Phys. Lett. A 368, 305 (2007).

[18] N.T Shawagfeh, J. Math. Phys. 34, 4364 (1993).

[19] J.-H. He, X.H. Wu, Chaos Solitons Fractals 29, 108 (2006).

[20] J.-H. He, Int. J. Mod. Phys. B 20, 1141 (2006).

[21] D.-H. Shou, J.H.-He, Phys. Lett. A 372, 233 (2008).

[22] S. Momani, Appl. Math. Comput. 173, 459 (2005).

[23] J. Rashidinia, R. Mohammadi, Int. J. Comput. Math. 85, 843 (2008). 